\title{
Australian sites of astronomical heritage
}

\author{
T. Stevenson ${ }^{1,2}$ and N. Lomb ${ }^{1}$ \\ ${ }^{1}$ Sydney Observatory, Powerhouse Museum, Sydney \\ email: toner.stevenson@phm.gov.au \\ ${ }^{2}$ The University of Sydney, Faculty of Arts, Museum Studies, NSW, Australia
}

\begin{abstract}
The heritage of astronomy in Australia has proven an effective communication medium. By interpreting science as a social and cultural phenomenon new light is thrown on challenges, such as the dispersal of instruments and problems identifying contemporary astronomy heritage. Astronomers are asked to take note and to consider the communication of astronomy now and in the future through a tangible heritage legacy.
\end{abstract}

"I was thrilled to be close to the historic 1874 large refracting lens telescope! The modern computer controlled reflecting telescope was interesting although it did not bring me an equal level of excitement as the 1874 telescope" commented a recent visitor to Sydney Observatory. Heritage can be a relevant, humanising and highly effective form of astronomy communication. In Australia astronomical heritage has encountered many obstacles including the inability of research organisations to identify and preserve important instruments. This includes the Yale Columbia Telescope remains from Mt Stromlo used for an artistic work.

Heritage collections are a medium through which scientists can communicate astronomy for the benefit of all. The legacy of astronomical results and methods is not confined to published papers. According to French sociologist, Bruno Latour, it is the instruments, the sites and even the workplaces of scientific endeavour that have agency in the production of the results. Furthermore historic objects have great potential to engage the public by presenting science within humanity through narratives of successes and failures. There is clear evidence for this at Sydney Observatory where bringing back the historic collection has helped to escalate visitor attendances in the past thirty years by a factor of eight.

Nineteenth century astronomers kept notebooks, correspondence, and well-used brass, polished glass and timber instruments. Unfortunately contemporary technology is not easily identifiable as heritage. Artefacts, such as the first Apple Computer and the Image Photon Counting System, from the Anglo Australian Telescope, are both innovative technology artefacts almost discarded in the past. These are two of the 100527 artefacts on the popular Powerhouse Museum collections database attracting over 900000 visits per year. In Australia, museums adopt the Significance 2.0 model for assessing collections. Its four primary criteria are: 'the historic; artistic; scientific and social or spiritual values that items and collections have for past, present and future generations.' There is a further four comparative criteria: 'provenance (who used it, where and when), rarity or representativeness, completeness and interpretive capacity'.

Astronomers need to carefully consider how and where their heritage remains will be kept, interpreted and displayed. Although museums cannot keep everything, using a sound methodology they can ensure that astronomy is represented in society. The future communication of astronomy through material culture depends on what astronomers do today. 
References: Powerhouse Museum Collections database: http://from.ph/157946; Significance 2.0: http://www.environment.gov.au/heritage/publications/significance2-0/ 